\title{
Improving seismic interpretation: a high-contrast approximation to the reflection coefficient of a plane longitudinal wave
}

\author{
Yin Xingyao*, Zong Zhaoyun and Wu Guochen \\ China University of Petroleum, Qingdao, Shandong 266580, China \\ (C) China University of Petroleum (Beijing) and Springer-Verlag Berlin Heidelberg 2013
}

\begin{abstract}
Linearized approximations of reflection and transmission coefficients set a foundation for amplitude versus offset (AVO) analysis and inversion in exploration geophysics. However, the weak properties contrast hypothesis of those linearized approximate equations leads to big errors when the two media across the interface vary dramatically. To extend the application of AVO analysis and inversion to high contrast between the properties of the two layers, we derive a novel nonlinearized high-contrast approximation of the PP-wave reflection coefficient, which establishes the direct relationship between PPwave reflection coefficient and $\mathrm{P}$-wave velocities, $\mathrm{S}$-wave velocities and densities across the interface. (A PP wave is a reflected compressional wave from an incident compressional wave (P-wave).) This novel approximation is derived from the exact reflection coefficient equation with Taylor expansion for the incident angle. Model tests demonstrate that, compared with the reflection coefficients of the linearized approximations, the reflection coefficients of the novel nonlinearized approximate equation agree with those of the exact PP equation better for a high contrast interface with a moderate incident angle. Furthermore, we introduce a nonlinear direct inversion method utilizing the novel reflection coefficient equation as forward solver, to implement the direct inversion for the six parameters including P-wave velocities, S-wave velocities, and densities in the upper and lower layers across the interface. This nonlinear inversion algorithm is able to estimate the inverse of the nonlinear function in terms of model parameters directly rather than in a conventional optimization way. Three examples verified the feasibility and suitability of this novel approximation for a high contrast interface, and we still could estimate the six parameters across the interface reasonably when the parameters in both media across the interface vary about $50 \%$.
\end{abstract}

Key words: High-contrast interface, reflection coefficient, amplitude variation with angle, multiparameter estimation, artificial neural network inversion

\section{Introduction}

The Zoeppritz equation (Zoeppritz and Erdbebnenwellen, 1919) and its approximations as the fundamental mathematical formulae for describing the amplitudes of $\mathrm{PP}$ reflected waves from $\mathrm{P}$-wave incident plane waves in exploration geophysics under plane wave approximation play an important role in AVO analysis/inversion (Smith and Gidlow, 1987; Buland and Omre, 2003; Downton and Lines, 2004; Yin et al, 2008; Zhang et al, 2012; Zong et al, 2012a; Zhang et al, 2013), lithology prediction (Ursin et al, 2003; Fu et al, 2005; Buland et al, 2008; Ulvmoen and Omre, 2010; Ulvmoen et al, 2010; Rimstad et al, 2012) and fluid discrimination (Zhang et al, 2010; Russell et al,

*Corresponding author. email: xyyin@upc.edu.cn Received June 20, 2013
2011; Rimstad et al, 2012; Wang et al, 2012; Zong et al, $2012 b$ ). The Zoeppritz equation gives the precise values of the amplitudes of the PP reflected plane wave. However, its intrinsic nonlinearity makes it less appropriate in practical applications. Therefore, linearized approximations with different parameterization of the Zoeppritz equations are more popular and practical (Bortfeld, 1961; Shuey, 1985; $\mathrm{Lu}$ and McMechan, 2004; Vedanti and Sen, 2009; Karimi et al, 2010; Alemie and Sacchi, 2011; Kim et al, 2011; Zhu and McMechan, 2012; Zong et al, 2012b). For details about different types of linearized approximations see Russell et al (2011). The linearized approximations are derived under the hypothesis of weak property contrasts between layers or limited incident angle. However, these assumptions do not hold especially at unconformities or at interfaces between different lithofacies (Ayzenberg et al, 2009; Skopintseva et al, 2011). Therefore, in this paper, we attempt to derive an approximation of the PP reflection coefficient to adjust to 
high contrast situations.

We utilize the forward modeling and nonlinear inversion method to test the feasibility and suitability of this novel approximation. Two forward modeling models with different degrees of property contrast are established and we compare the reflection coefficients with the novel approximation, exact Zeoppritz equation and linearized approximation, respectively. As for the inversion method, an artificial neural network nonlinear direct inversion is introduced to estimate the six parameters with the novel approximation as a forward solver. The artificial neural network nonlinear inversion was initially proposed by Rủžek et al (2009). It is a kind of nonlinear direct inversion approach rather than an optimization approach. It has been proved that inversion is more efficient than optimization when attempting to find the inverse of $G(\cdot)$, which is the forward solver. It can provide several solutions like the multiple realizations in stochastic inversion by Bayesian inference (Buland and Omre, 2003). For details about this inversion method refer to Rủžek et al (2009). In appendix A hereunder, we will give the necessary description of this method for the nonlinear inversion problem with the novel approximation equation.

\section{Modeling}

The general theory of the P-wave reflection has been widely discussed in the literature, so we shall reproduce here only that required for an understanding of the notation and terminology that we will use in this paper. For the cases of incident longitudinal waves polarized in the plane of incidence, the reflection coefficient of longitudinal waves can be expressed as (Aki and Richards, 1980),

$$
R_{\mathrm{PP}}=\left(E^{-} F^{+}-G^{+} H^{-} p^{2}\right) / D
$$

with

$$
\begin{aligned}
& E^{+}=b \frac{\cos \theta_{\mathrm{P} 1}}{V_{\mathrm{P} 1}}+c \frac{\cos \theta_{\mathrm{P} 2}}{V_{\mathrm{P} 2}} \quad E^{-}=b \frac{\cos \theta_{\mathrm{P} 1}}{V_{\mathrm{P} 1}}-c \frac{\cos \theta_{\mathrm{P} 2}}{V_{\mathrm{P} 2}} \\
& F^{+}=b \frac{\cos \theta_{\mathrm{S} 1}}{V_{\mathrm{S} 1}}+c \frac{\cos \theta_{\mathrm{S} 2}}{V_{\mathrm{S} 2}} \quad G^{+}=a+d \frac{\cos \theta_{\mathrm{P} 1}}{V_{\mathrm{P} 1}} \frac{\cos \theta_{\mathrm{S} 2}}{V_{\mathrm{S} 2}} \\
& G^{-}=a-d \frac{\cos \theta_{\mathrm{P} 1}}{V_{\mathrm{P} 1}} \frac{\cos \theta_{\mathrm{S} 2}}{V_{\mathrm{S} 2}} \quad H^{-}=a-d \frac{\cos \theta_{\mathrm{P} 2}}{V_{\mathrm{P} 2}} \frac{\cos \theta_{\mathrm{S} 1}}{V_{\mathrm{S} 1}} \\
& D=E^{+} F^{+}+G^{-} H^{-} p^{2} \quad a=\rho_{2}\left(1-2 V_{\mathrm{S} 2}^{2} p^{2}\right)-\rho_{1}\left(1-2 V_{\mathrm{S} 1}^{2} p^{2}\right) \\
& b=\rho_{2}\left(1-2 V_{\mathrm{S} 2}^{2} p^{2}\right)+2 \rho_{1} V_{\mathrm{S} 1}^{2} p^{2} \quad c=\rho_{1}\left(1-2 V_{\mathrm{S} 1}^{2} p^{2}\right)+2 \rho_{1} V_{\mathrm{S} 1}^{2} p^{2} \\
& d=2 \rho_{2} V_{\mathrm{S} 2}^{2}-2 \rho_{1} V_{\mathrm{S} 1}^{2} \quad \frac{\sin \theta_{\mathrm{P} 1}}{V_{\mathrm{P} 1}}=\frac{\sin \theta_{\mathrm{S} 1}}{V_{\mathrm{S} 1}}=\frac{\sin \theta_{\mathrm{P} 2}}{V_{\mathrm{P} 2}}=\frac{\sin \theta_{\mathrm{S} 2}}{V_{\mathrm{S} 2}}=p
\end{aligned}
$$

where $\theta_{\mathrm{P} 1}, \theta_{\mathrm{S} 1}, \theta_{\mathrm{P} 2}$ and $\theta_{\mathrm{S} 2}$ are the incident angle of the longitudinal wave, the reflective angle of the shear wave, and the transmission angles of the longitudinal wave and shear wave, respectively; $R_{\mathrm{PP}}$ is the reflection coefficient of the longitudinal wave; $V_{\mathrm{P} 1}, V_{\mathrm{S} 1}$ and $\rho_{1}$ are the P-wave velocity, S-wave velocity and density in medium 1 , and $V_{\mathrm{P} 2}, V_{\mathrm{S} 2}$ and $\rho_{2}$ are the same in medium $2 ; \rho$ is the ray parameter.

The linearized approximation of Eq. (1) is given by Aki and Richards (1980) as,

$$
R_{\mathrm{PP}}=\frac{1}{2}\left(1-4 V_{\mathrm{S}}^{2} p^{2}\right) \frac{\Delta \rho}{\rho}+\frac{1}{2 \cos ^{2} \theta_{\mathrm{P}}} \frac{\Delta V_{\mathrm{P}}}{V_{\mathrm{P}}}-4 V_{\mathrm{S}}^{2} p^{2} \frac{\Delta V_{\mathrm{S}}}{V_{\mathrm{S}}}
$$

where $\theta_{\mathrm{P}}$ is incident angle, $\Delta V_{\mathrm{P}} / V_{\mathrm{P}}$ is $\mathrm{P}$-wave velocity reflectivity, $\Delta V_{\mathrm{S}} / V_{\mathrm{S}}$ is $\mathrm{S}$-wave velocity reflectivity and $\Delta \rho / \rho$ is density reflectivity, and they can be expressed as,

$$
\begin{aligned}
& \frac{\Delta V_{\mathrm{P}}}{V_{\mathrm{P}}}=\frac{2\left(V_{\mathrm{P} 1}-V_{\mathrm{P} 2}\right)}{\left(V_{\mathrm{P} 1}+V_{\mathrm{P} 2}\right)} \\
& \frac{\Delta V_{\mathrm{S}}}{V_{\mathrm{S}}}=\frac{2\left(V_{\mathrm{S} 1}-V_{\mathrm{S} 2}\right)}{\left(V_{\mathrm{S} 1}+V_{\mathrm{S} 2}\right)} \\
& \frac{\Delta \rho}{\rho}=\frac{2\left(\rho_{1}-\rho_{2}\right)}{\left(\rho_{1}+\rho_{2}\right)}
\end{aligned}
$$

Taking the Taylor expansion for incident angle of Eq. (1), we can express $R_{\mathrm{Pp}}$ in a closed form as,

$$
\begin{aligned}
& R_{\mathrm{PP}}(\theta)=\frac{\theta^{2}}{A_{1} A_{2} V_{\mathrm{P} 1}}\left(V_{\mathrm{P} 1} V_{\mathrm{P} 2}^{2} V_{\mathrm{S} 1} \rho_{1} \rho_{2}^{2}+V_{\mathrm{P} 1} V_{\mathrm{P} 2}^{2} V_{\mathrm{S} 2} \rho_{2}^{3}+\right. \\
& V_{\mathrm{P} 2}^{3} V_{\mathrm{S} 1} \rho_{1}^{2} \rho_{2}+V_{\mathrm{P} 2}^{3} V_{\mathrm{S} 2} \rho_{1} \rho_{2}^{2}-2 V_{\mathrm{P} 1} V_{\mathrm{P} 2}^{2} V_{\mathrm{S} 1} V_{\mathrm{S} 2} \rho_{1}^{2} \rho_{2}+4 V_{\mathrm{P} 2}^{2} V_{\mathrm{S} 1} V_{\mathrm{S} 2} \rho_{1} \rho_{2}^{2} \\
& -2 V_{\mathrm{P} 2}^{2} V_{\mathrm{S} 1} V_{\mathrm{S} 2} \rho_{2}^{3}+8 V_{\mathrm{P} 2} V_{\mathrm{S} 1}^{3} \rho_{1}^{2} \rho_{2}+8 V_{\mathrm{P} 2} V_{\mathrm{S} 1}^{2} V_{\mathrm{S} 2} \rho_{1}^{2} \rho_{2}-8 V_{\mathrm{P} 2} V_{\mathrm{S} 1} V_{\mathrm{S} 2}^{2} \rho_{1} \rho_{2}^{2} \\
& \left.+8 V_{\mathrm{S} 1}^{4} \rho_{1}^{3}-16 V_{\mathrm{S} 1}^{2} V_{\mathrm{S} 2}^{2} \rho_{1}^{2} \rho_{2}+8 V_{\mathrm{S} 2}^{4} \rho_{1} \rho_{2}^{2}-A_{1} V_{\mathrm{P} 1} V_{\mathrm{P} 2} \rho_{2}\right)+\frac{2 V_{\mathrm{P} 2} \rho_{2}}{A_{2}}-1
\end{aligned}
$$

with

$$
\begin{aligned}
& A_{1}=V_{\mathrm{P} 2} V_{\mathrm{S} 2} \rho_{2}^{2}+V_{\mathrm{P} 1} V_{\mathrm{S} 2} \rho_{1} \rho_{2}+V_{\mathrm{P} 1} V_{\mathrm{S} 1} \rho_{1}^{2}+V_{\mathrm{P} 2} V_{\mathrm{S} 1} \rho_{1} \rho_{2} \\
& A_{2}=V_{\mathrm{P} 1} \rho_{1}+V_{\mathrm{P} 2} \rho_{2}
\end{aligned}
$$

To examine the accuracy of our novel approximation of PP-wave reflectivity, we compare the analytical result of $R_{\mathrm{PP}}$ with the exact equation (1), linearized approximation equation (2) and our novel nonlinearized approximation equation (6) with the two-layer models in Table 1. Model one represents the weak property contrast case, and the model two represents the high contrast situation. Fig. 1 displays the reflection coefficient of the model one with the exact equation (1) (solid black), linearized approximation equation (2) (dashed blue) and our novel nonlinearized approximation equation (6) (red dots), and we can see that reflection coefficients with these three equations show good similarity in the weak contrast case. Fig. 2 displays the result of the model two, we can see that the reflection coefficients from linearized approximation show high errors compared to that from the exact equation, and the reflection coefficient from our novel approximation still shows high similarity to that from the exact equation at a moderate incident angle.

\section{Nonlinear inversion}

To test the possibility of estimating parameters with our novel approximation, we introduce an artificial neural network direct nonlinear inversion method (Růžek et al, 2009). The inversion is formulated in a direct inversion scheme utilizing Eq. (6) as the forward solver. We restrict our computational domain to various two-layer models to test the effectiveness and potential of our novel approximation in high contrast media. Similar to Rabben et al (2008), we attempt to 
Table 1 Model parameters for forward modeling

Model One

\begin{tabular}{cccc}
\hline Parameters & $\begin{array}{c}\text { P-wave velocity } \\
\mathrm{m} / \mathrm{s}\end{array}$ & $\begin{array}{c}\text { S-wave velocity } \\
\mathrm{m} / \mathrm{s}\end{array}$ & Density, $\mathrm{kg} / \mathrm{m}^{3}$ \\
\hline Upper medium & 2898 & 1610 & 2424 \\
Lower medium & 2857 & 1666 & 2375 \\
\hline
\end{tabular}

Model Two

\begin{tabular}{cccc}
\hline Parameters & $\begin{array}{c}\text { P-wave velocity } \\
\mathrm{m} / \mathrm{s}\end{array}$ & $\begin{array}{c}\text { S-wave velocity } \\
\mathrm{m} / \mathrm{s}\end{array}$ & Density, $\mathrm{kg} / \mathrm{m}^{3}$ \\
\hline Upper medium & 2898 & 1290 & 2424 \\
Lower medium & 2557 & 1666 & 2075 \\
\hline
\end{tabular}

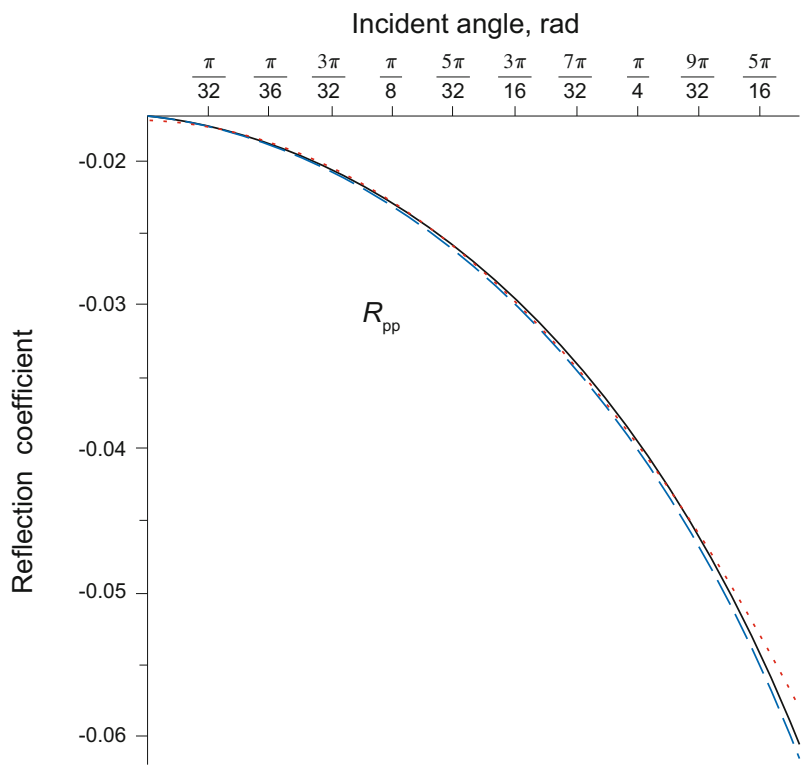

Fig. 1 Reflection coefficient comparison between the exact equation (1) (solid black), linearized approximation equation (2) (dashed blue) and our novel nonlinearized approximation equation (6) (red dots) with the model one in Table 1

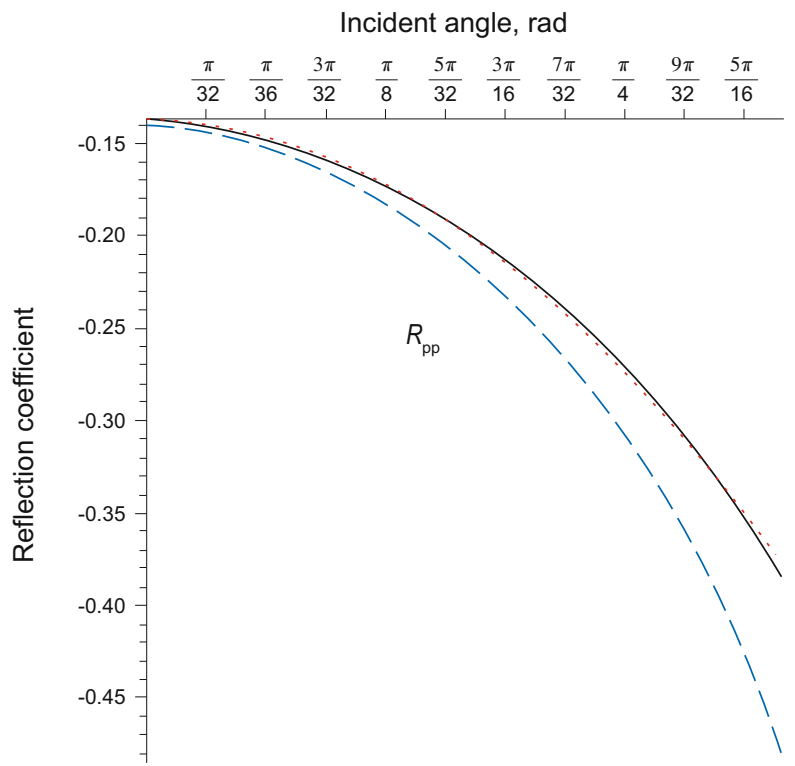

Fig. 2 Reflection coefficient comparison with the exact equation (1) (solid black), linearized approximation equation (2) (dashed blue) and our novel nonlinearized approximation equation (6) (red dots) with the model two in Table 1 estimate the parameters from reflection coefficients to avoid the wavelet estimation and the convolution in the modeling.

We utilized the exact equation (1) as the synthetic model. The model vector $\mathbf{m}$ comprises of $\mathrm{P}$-wave velocities, $\mathrm{S}$-wave velocities and densities in the upper and lower layers. The observed data $\mathbf{d}$ are the reflection coefficients with different incident angles of Eq. (1).

The model parameters and the data parameters can be related through the nonlinear forward mapping,

$$
\mathbf{d}=G(\mathbf{m})
$$

Here, we attempt to search for all possible solutions of model parameters to satisfy the observed reflection coefficients. With the artificial neural network direct inversion method, we suppose the inverse to $G(\mathbf{m})$ is $G^{-1}(\mathbf{d})$. Although the inverse mapping may not exist in entire spaces, we can restrict ourselves to sufficiently small joint subspaces, inside which the mapping $G^{-1}(\cdot)$ is so smooth that the inverse of $G$ does exist,

$$
G^{-1}\left(\mathbf{d}_{0}\right)=\mathbf{m}_{0}
$$

The artificial neural network direct inversion method is an inverse (not optimizing) algorithm, utilizing numerical approximation of Eq. (8) in empirically constrained subspaces. Supposing there exists an inverse mapping and its numerical approximation inside these subspaces, it works simultaneously with a population of several so-called individuals. Each individual contains a parameter vector, a data vector and the model error. The model error is used for relative classification of distinct models within the population and for their sorting from the best to the worst model. The computation records already evaluated and tested models so that these models can be reused later. Repeated usage of some models generates the possibility of efficient inversion with minimum number of forward evaluations. Besides, several solutions can be expected with this algorithm. Details of this algorithm can be seen in Růžek et al (2009). We shall reproduce the algorithm in Appendix A but only to a level required for an understanding of the notation and terminology that we use in our examples and discussion section.

\section{Examples}

Various two-layer models are established in the inversion test. The first one is a gas sand/shale model. The P-wave velocity $\left(V_{\mathrm{P} 1}\right)$, S-wave velocity $\left(V_{\mathrm{S} 1}\right)$ and density (Density 1) in the gas sand is $2,438 \mathrm{~m} / \mathrm{s}, 1,625 \mathrm{~m} / \mathrm{s}$ and $2,140 \mathrm{~kg} / \mathrm{m}^{3}$, respectively, while the $\mathrm{P}$-wave velocity $\left(V_{\mathrm{P} 2}\right), \mathrm{S}$-wave velocity $\left(V_{\mathrm{S} 2}\right)$ and density (Density 2 ) in the shale sand is $3,048 \mathrm{~m} / \mathrm{s}$, $1,244 \mathrm{~m} / \mathrm{s}$ and $2,400 \mathrm{~kg} / \mathrm{m}^{3}$, respectively, and we refer to this as the "first" model. The relative variation ratio of parameters in two layers of the first model is displayed in Fig. 3. For this model, Eq. (1) is used to generate $R_{\mathrm{PP}}$ at different incident angles to simulate observed data. The introduced nonlinear inversion method is then used to generate ten solutions for each of the six parameters. With the introduced inversion method, the results of parameters estimation are displayed 
in Fig. 4. Taking $V_{\mathrm{P} 1}$ for example, the left figure shows the comparison between the estimated solutions and the true value. The sequence numbers from 1 to 10 indexes the ten solutions for $V_{\mathrm{P} 1}$, the value at sequence number 11 gives the average value of the ten estimated values, and the value at sequence number 12 gives the true value. The right figure displays the relative error between the estimated solutions and the true value. The sequence numbers from 1 to 10 indexes the relative error between each estimated solution for $V_{\mathrm{P} 1}$ and the true value, sequence number 11 gives the relative error between the average value of all ten values and the true value. The same key applies to the other five parameters. From Fig. 4 , we can see that all six parameters can be inverted well and the relative error is around 3\% for each parameter.

Fig. 5 displays the relative change rate of the parameters from medium 1 to medium 2 in the second model. It shows that the parameters in medium 2 vary around $50 \%$ with respect to the parameters in medium 1. Fig. 6 displays the result of estimating six parameters with the second model

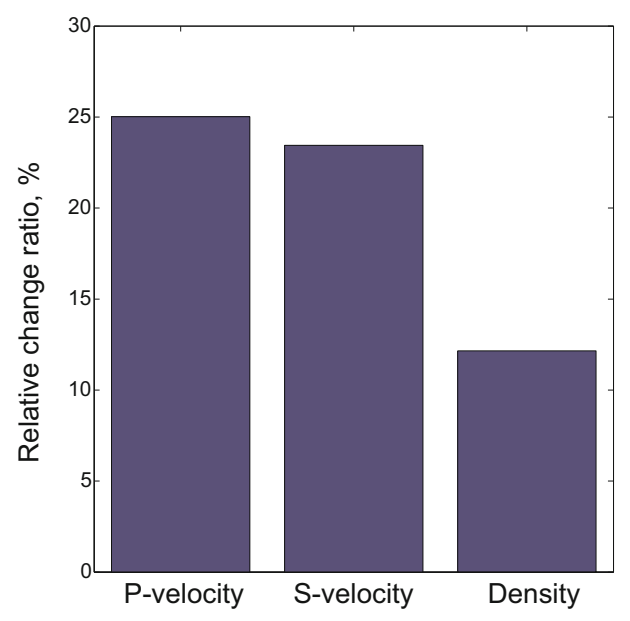

Fig. 3 Relative variation ratio of parameters in two layers with the first model
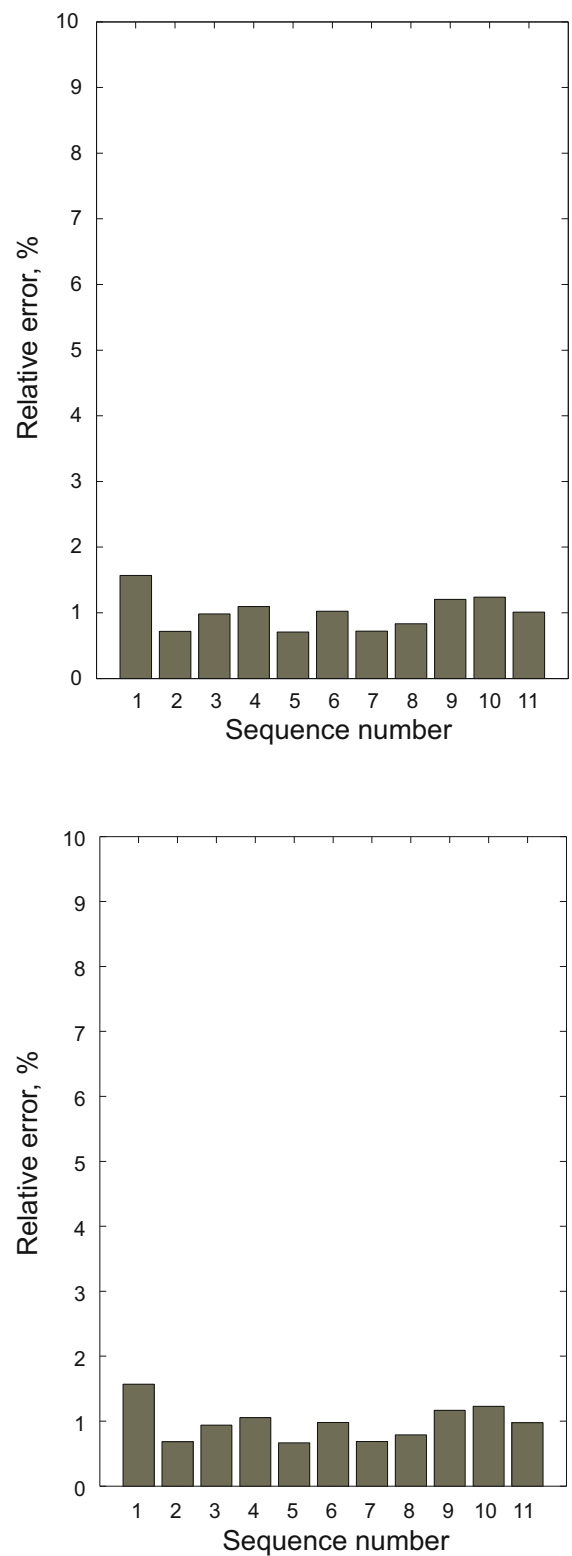
(Continued)
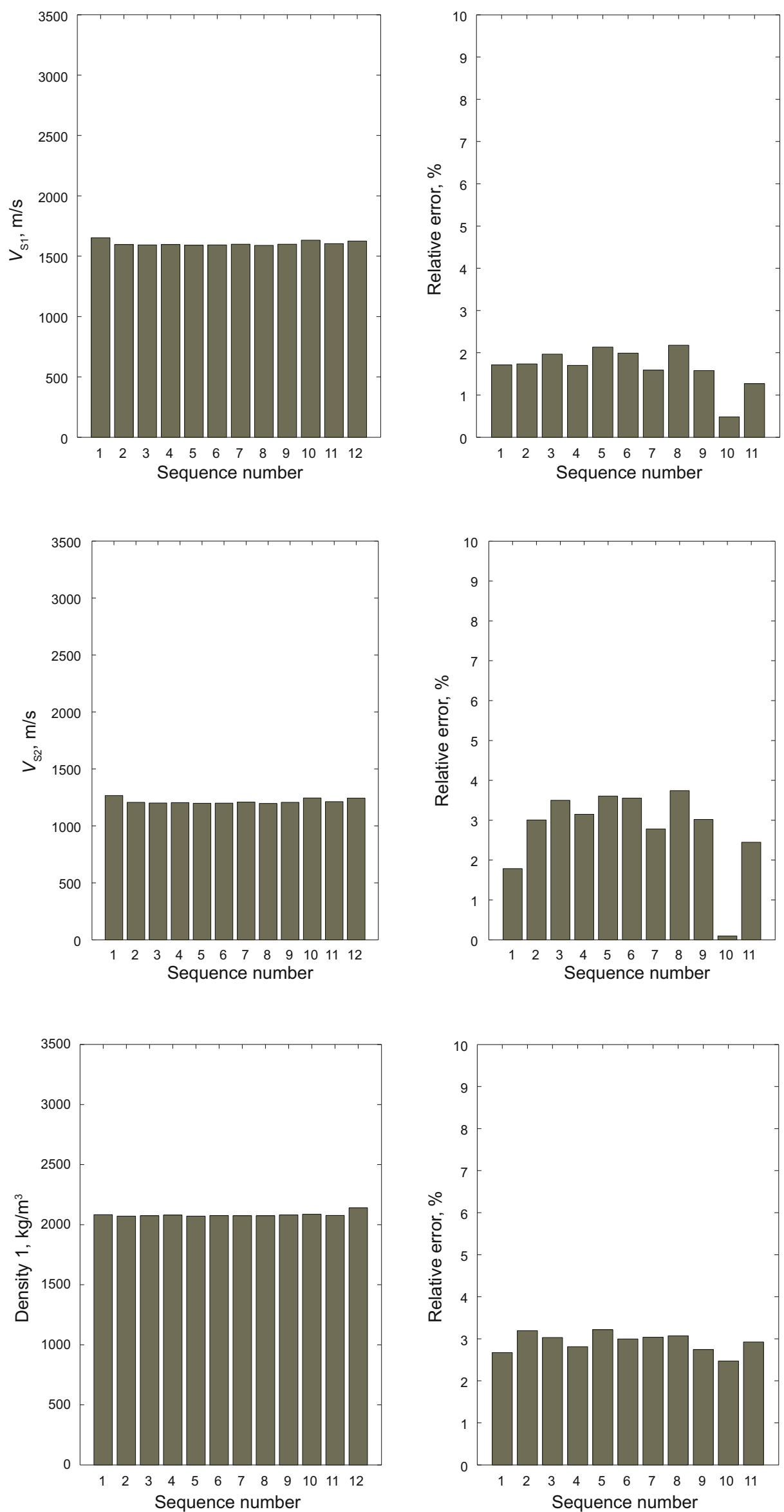
(Continued)
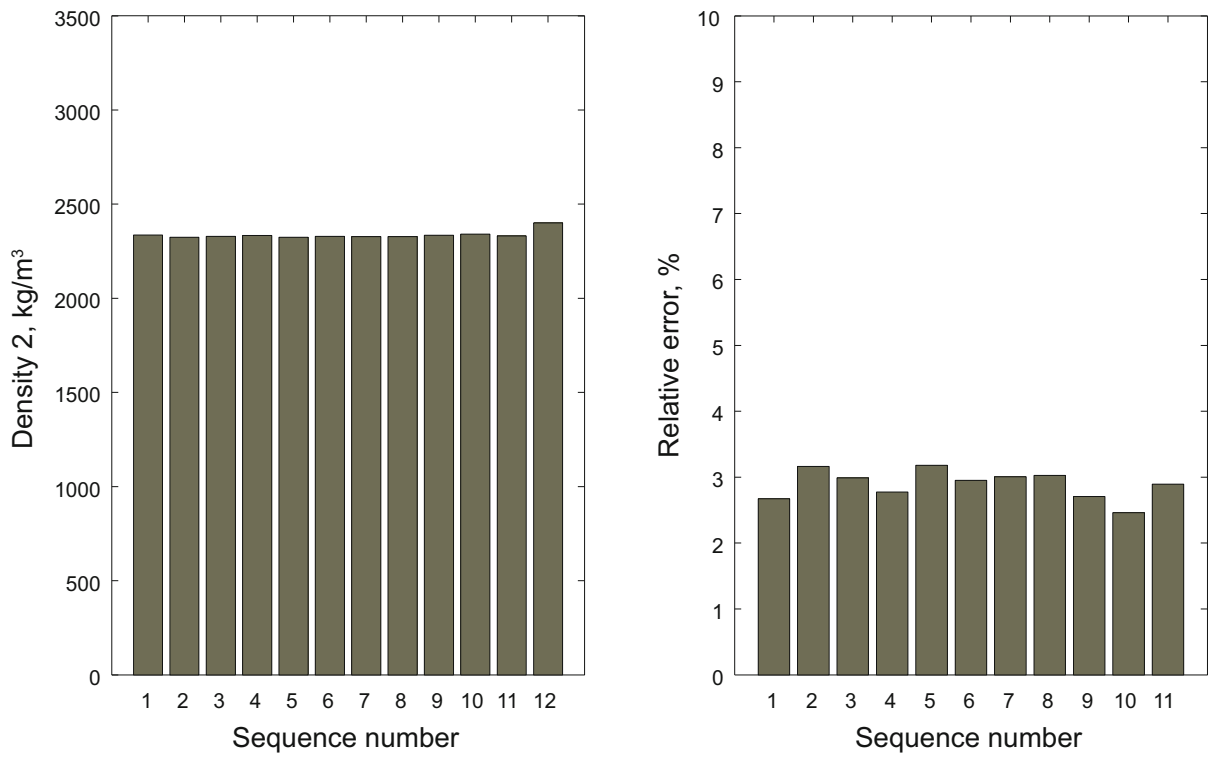

Fig. 4 Results of estimating six parameters with the first model

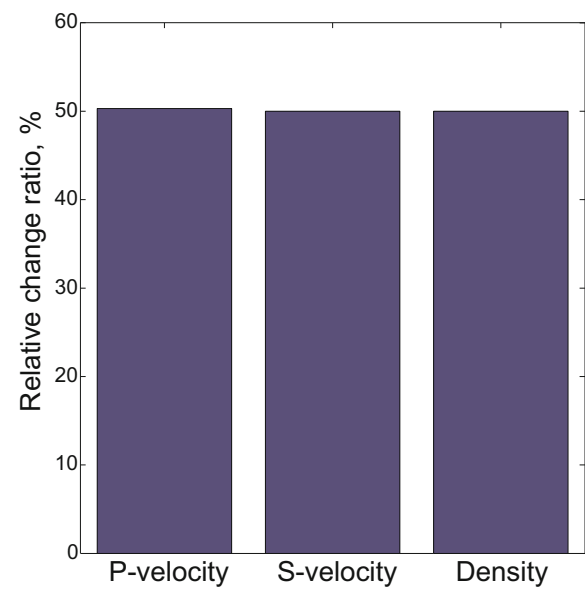

Fig. 5 Relative variation ratios of parameters in two layers with the second model

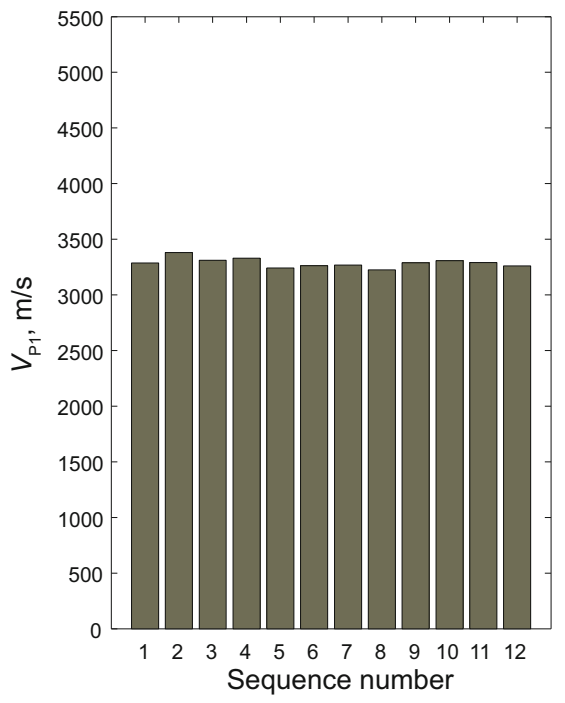

when the maximum incident angle is 31 degree. We can see that even in high contrast media, the inversion with the exact reflection coefficient equation still estimates the six parameters reasonably.

Fig. 7 to Fig. 12 display the inversion results with the 2-D surface model. Taking the $\mathrm{P}$-wave velocity in upper medium for example, Fig. 7(a) - 7(c) display P-wave velocity in upper medium of the true model, inverted result and the relative error between the true model and inverted result, respectively. The same key applies to the other five parameters, as shown in Fig. 7 to Fig. 12. From the inverted results, we can see that, with the high-contrast approximation and the nonlinear inversion algorithm, we can obtain reasonable inversion results, and the relative error is around $4 \%$ for each parameter.

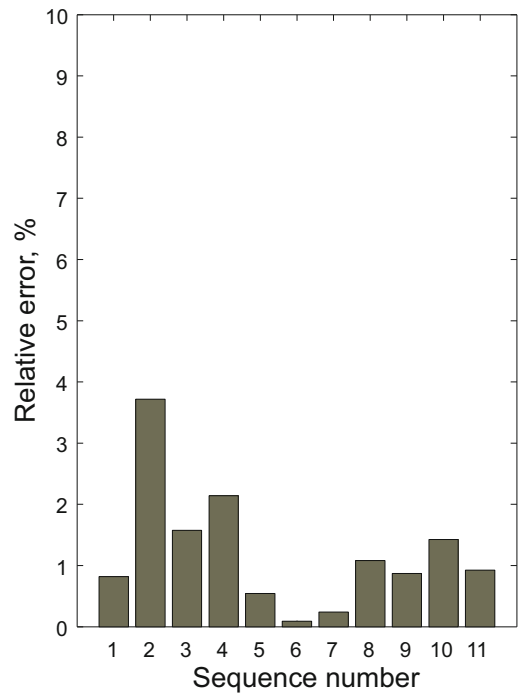

(To be continued) 
(Continued)
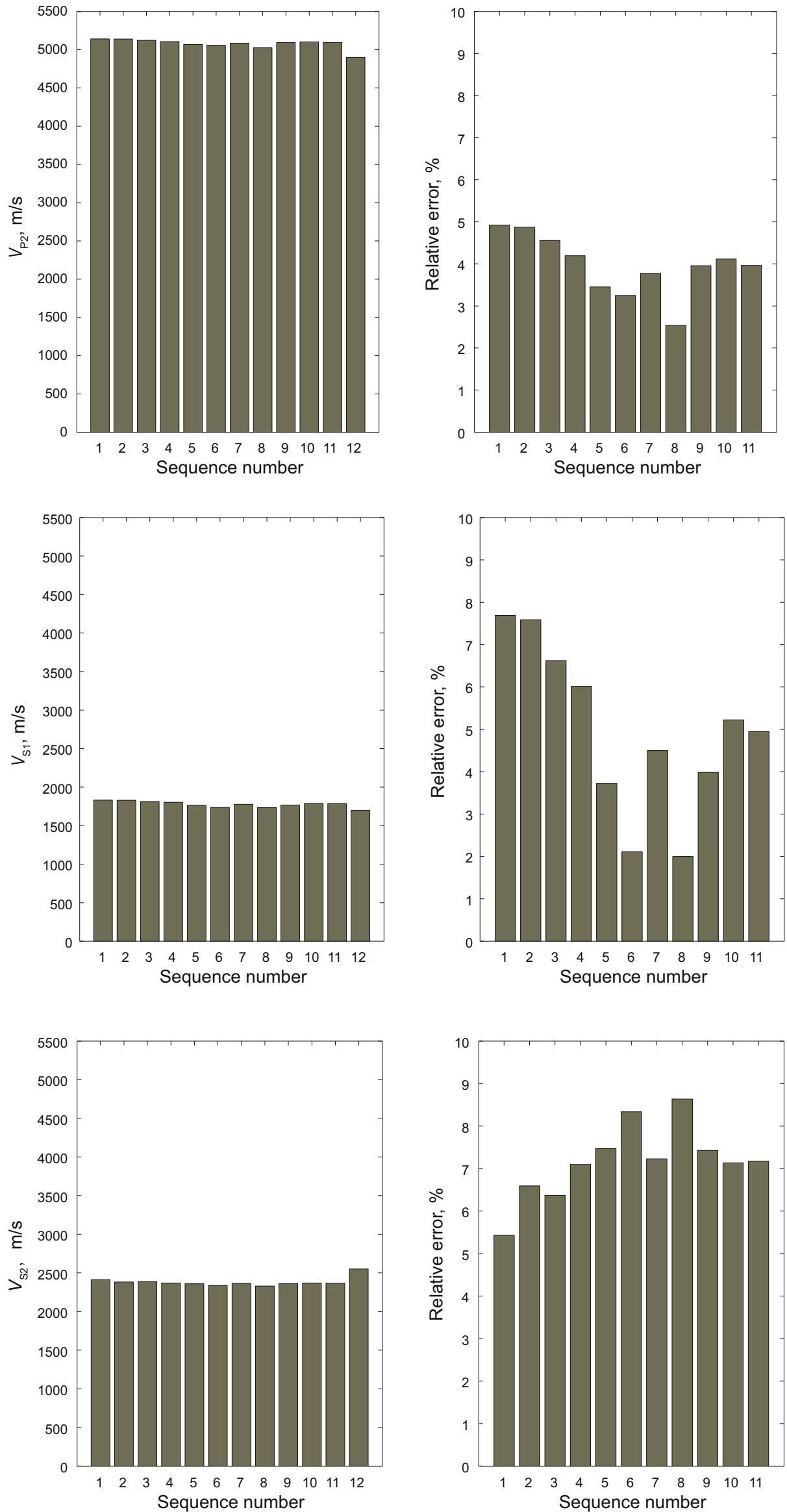
(Continued)
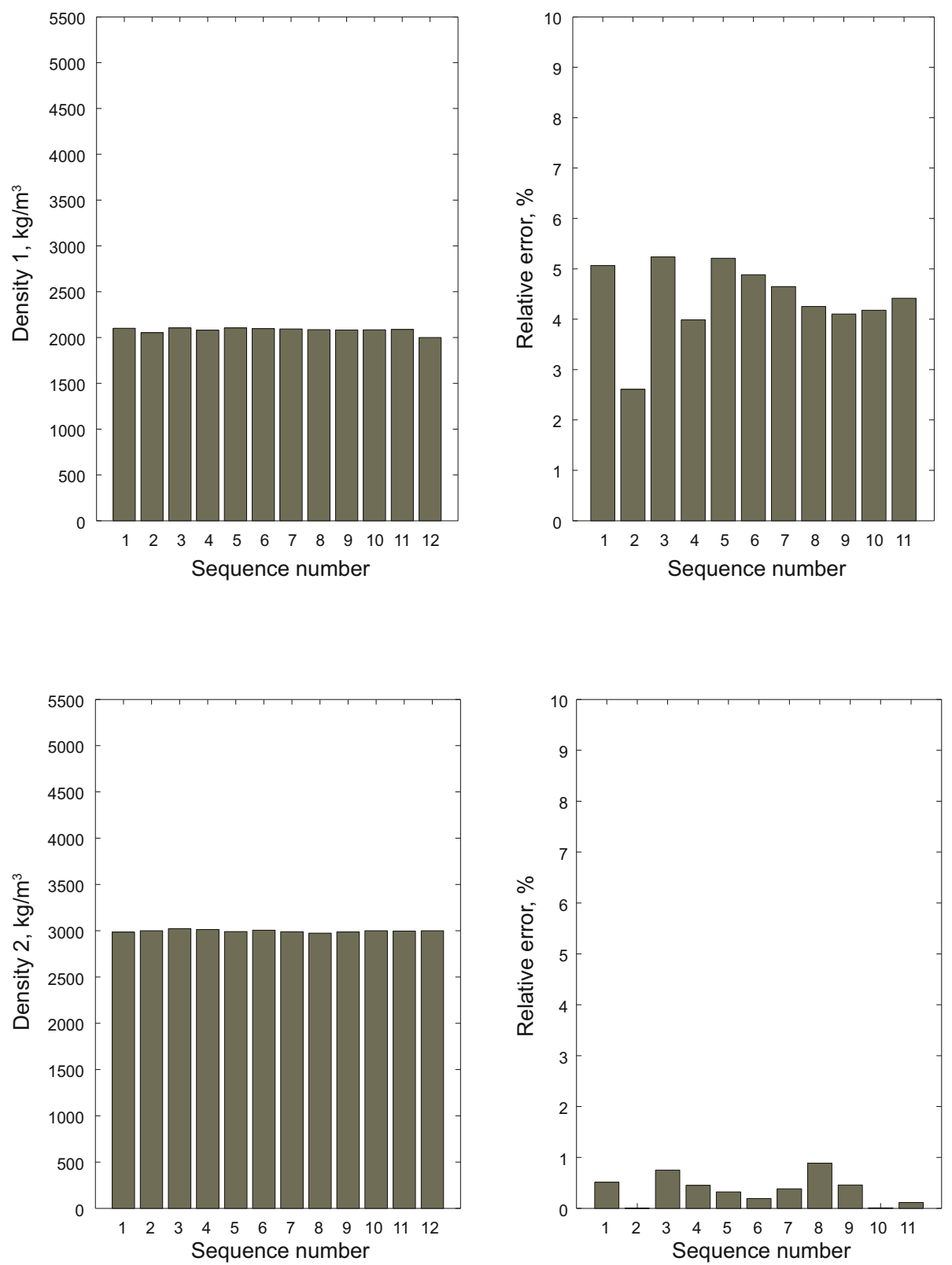

Fig. 6 Results of estimating six parameters with the second model (The description and definition of the figure are the same as in Fig. 4)

(a)

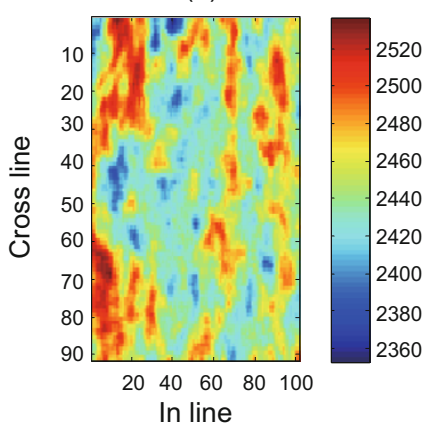

(b)

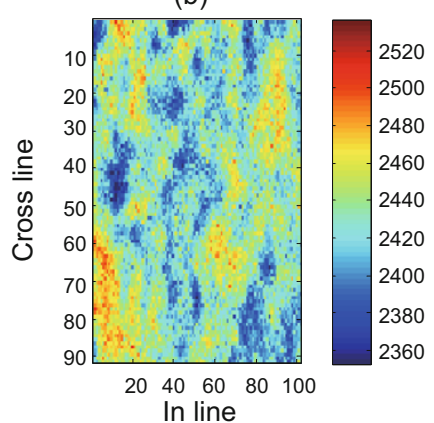

(c)

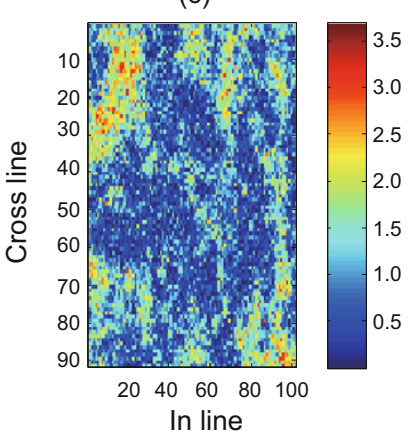

Fig. 7 P-wave velocities in the upper medium (a) True model, (b) Inverted result, and (c) Relative error 
(a)

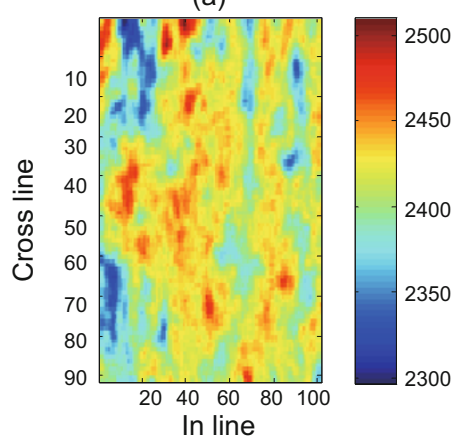

(b)

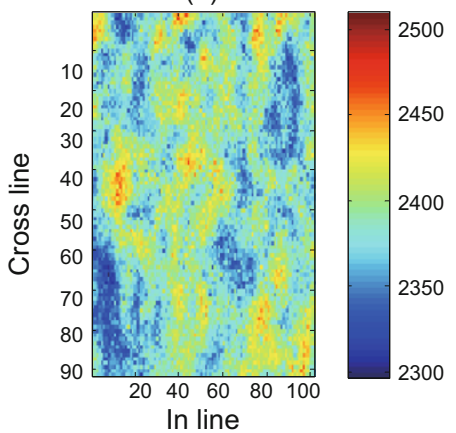

(c)

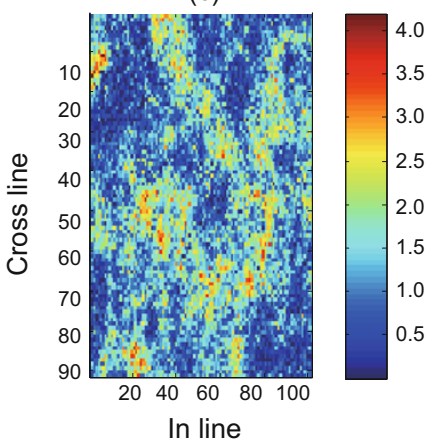

Fig. 8 P-wave velocities in the lower medium (a) True model, (b) Inverted result, and (c) Relative error

(a)

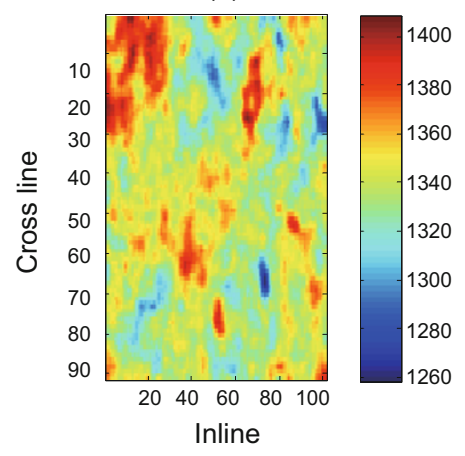

(b)

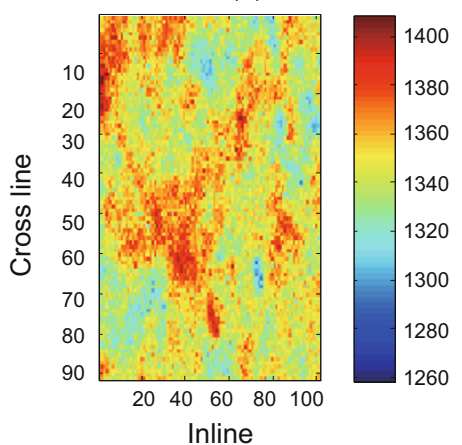

(c)

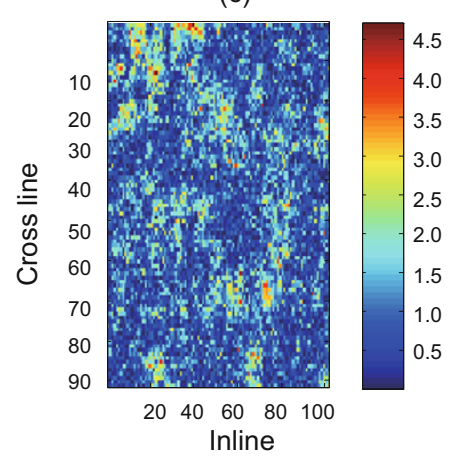

Fig. 9 S-wave velocities in the upper medium (a) True model, (b) Inverted result, and (c) Relative error

(a)

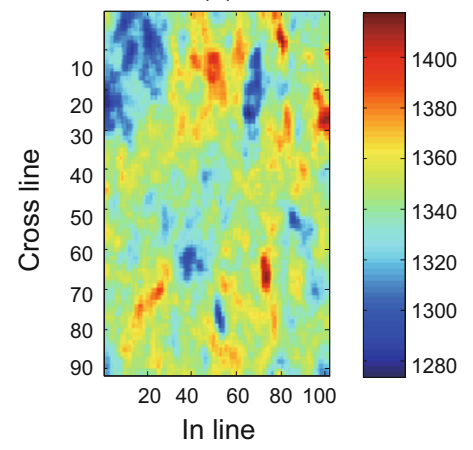

(b)

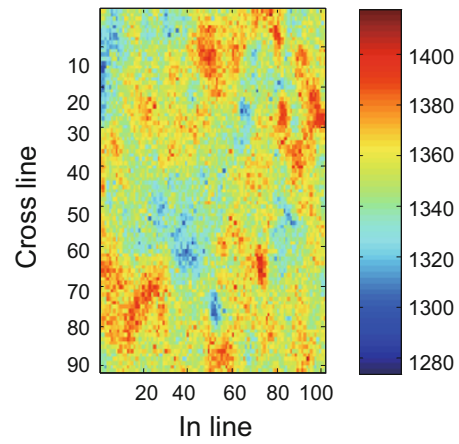

(c)

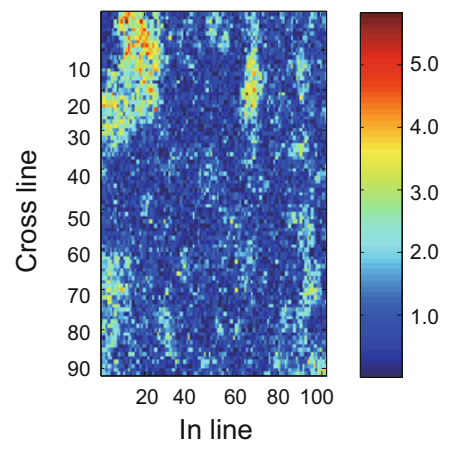

Fig. $10 \mathrm{~S}$-wave velocities in the lower medium (a) True model, (b) Inverted result, and (c) Relative error

(a)

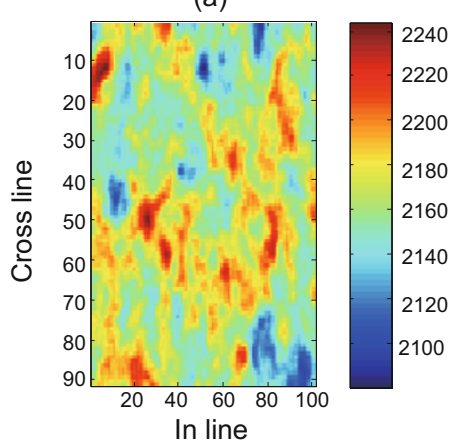

(b)

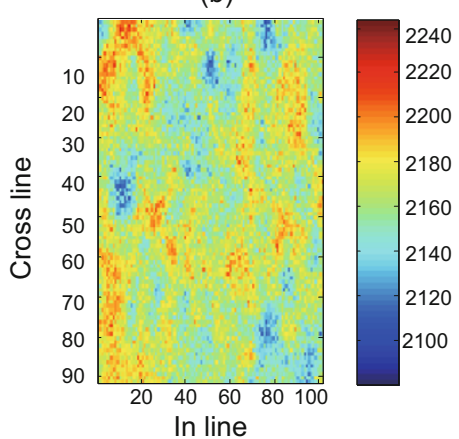

(c)

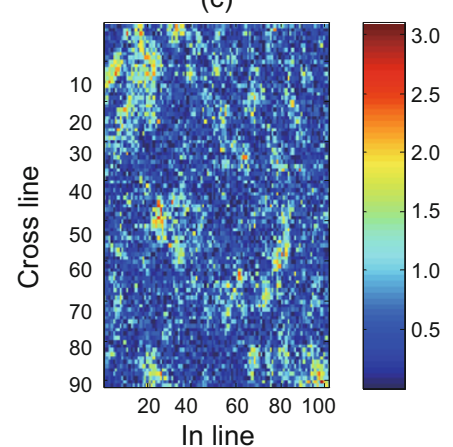

Fig. 11 Densities in the upper medium (a) True model, (b) Inverted result, and (c) Relative error 
(a)

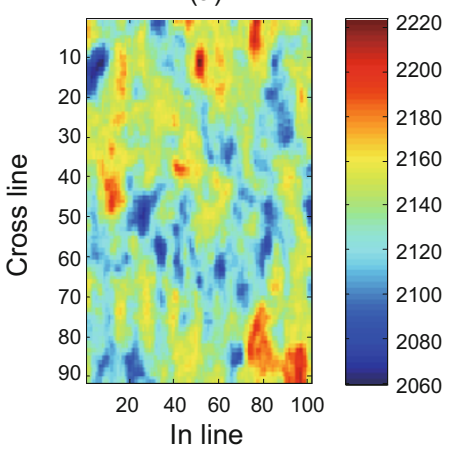

(b)

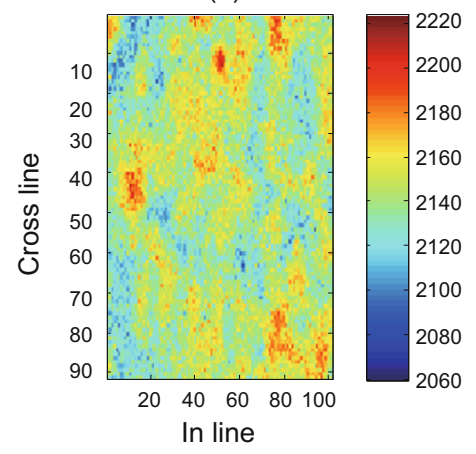

(c)

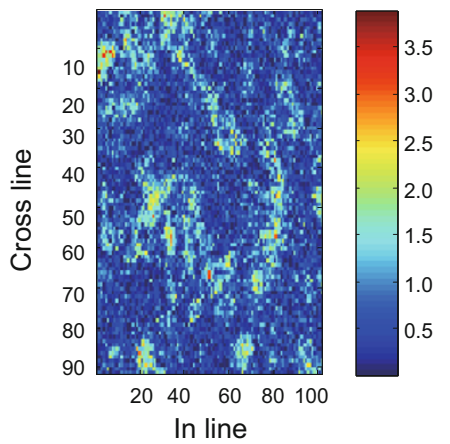

Fig. 12 Densities in the lower medium (a) True model, (b) Inverted result, and (c) Relative error

\section{Conclusions}

In this paper, we derived a high-contrast approximation of the exact PP reflection coefficient in terms of six parameters including P-wave velocities, S-wave velocities and densities in upper and lower layers around a reflector. We utilized the forward modeling and inversion method to test the validity and feasibility of this novel approximation. Forward modeling tests demonstrated the priority of the novel approximation to the linearized approximation in reflection coefficient modeling. A nonlinear direct inversion method was introduced to estimate the six layer parameters around a reflector. Model test showed that the inversion method for multi-parameters with our novel nonlinearized approximation of exact reflection coefficient equation could still get reasonable inversion result even when the parameters in both layers across the interface varied about $50 \%$.

\section{Acknowledgements}

We would like to acknowledge the sponsorship of the National 973 Program of China (2013CB228604) and the National Grand Project for Science and Technology (2011ZX05030-004-002,2011ZX05019-003 and 2011ZX05006-002) for funding this research. We also acknowledge the support of the Australian and Western Australian Governments and the North West Shelf Joint Venture Partners, as well as the Western Australian Energy Research Alliance (WA:ERA), and Foundation from Geophysical Key Lab of SINOPEC (WTYJYWX2013-04-01).

\section{References}

Aki K and Richards P G. Quantitative Seismology. 2nd Edition. W. H. Freeman and Co. 1980

Alemie W and Sacchi M D. High-resolution three-term AVO inversion by means of a Trivariate Cauchy probability distribution. Geophysics. 2011. 76(3): R43-R55

Ayzenberg M, Tsvankin I, Aizenberg A, et al. Effective reflection coefficients for curved interfaces in transversely isotropic media. Geophysics. 2009. 74(5): WB33-WB53

Bortfeld R. Approximations to the reflection and transmission coefficients of plane longitudinal and transverse waves. Geophysical Prospecting. 1961. 9(4): 485-502
Buland A and Omre H. Bayesian linearized AVO inversion. Geophysics. 2003. 68(1): 185-198

Buland A, Kolbjørnsen O, Hauge R, et al. Bayesian lithology and fluid prediction from seismic prestack data. Geophysics. 2008. 73(3): C13-C21

Downton J E and Lines L R. Three term AVO waveform inversion. SEG Technical Program Expanded Abstracts. 2004

$\mathrm{Fu}$ L, Wang J M, Cui F L, et al. 3D AVO analysis for identifying thin continental sandstone interbeds and deep volcanic rocks. Applied Geophysics. 2005. 2(1): 41-45

Karimi O, Omre H and Mohammadzadeh M. Bayesian closed-skew Gaussian inversion of seismic AVO data for elastic material properties. Geophysics. 2010. 75(1): R1-R11

Kim J S, Kim W K, Ha H S, et al. AVO analysis using crossplot and amplitude polynomial methods for characterisation of hydrocarbon reservoirs. Exploration Geophysics. 2011. 42(1): 25-41

$\mathrm{Lu} \mathrm{S}$ and McMechan G A. Elastic impedance inversion of multichannel seismic data from unconsolidated sediments containing gas hydrate and free gas. Geophysics. 2004. 69(1): 164-179

Rabben T E, Tjelmeland $\mathrm{H}$ and Ursin B. Nonlinear Bayesian joint inversion of seismic reflection coefficients. Geophysical Journal International. 2008. 173(1): 265-280

Rimstad K, Avseth P and Omre H. Hierarchical Bayesian lithology/ fluid prediction: a North Sea case study. Geophysics. 2012. 77(2): B69-B85

Russell B H, Gray D and Hampson D P. Linearized AVO and poroelasticity. Geophysics. 2011. 76(3): C19-C29

Růžek B P, Kolář P and Kvasnička M. Robust solver of a system of nonlinear equations. Technical Computing Prague. ISBN 978-807080-733-0. Praha. 2009. p.90

Shuey R T. A simplification of the Zoeppritz equations. Geophysics. 1985. 50(4): 609-614

Skopintseva L, Ayzenberg M, Landrø M, et al. Long-offset AVO inversion of PP reflections from plane interfaces using effective reflection coefficients. Geophysics. 2011. 76(6): C65-C79

Smith G C and Gidlow P M. Weighted stacking for rock property estimation and detection of gas. Geophysical Prospecting. 1987. 35(9): 993-1014

Ulvmoen M and Omre H. Improved resolution in Bayesian lithology/ fluid inversion from prestack seismic data and well observations: Part 1-Methodology. Geophysics. 2010. 75(2): R21-R35

Ulvmoen M, Omre H and Buland A. Improved resolution in Bayesian lithology/fluid inversion from prestack seismic data and well observations: Part 2-Real case study. Geophysics. 2010. 75(2): B73-B82

Ursin B, Bauer C, Zhao H S, et al. Combined seismic inversion and gravity modeling of a shallow anomaly in the southern Barents Sea. 
Geophysics. 2003. 68(4): 1140-1149

Vedanti N and Sen M K. Seismic inversion tracks in situ combustion: A case study from Balol oil field, India. Geophysics. 2009. 74(4): B103-112

Wang H Y, Sun Z D, Wang D, et al. Frequency-dependent velocity prediction theory with implication for better reservoir fluid prediction. The Leading Edge. 2012. 31(2): 160-166

Yin X Y, Yang P J and Zhang G Z. A novel prestack AVO inversion and its application. SEG Technical Program Expanded Abstracts. 2008

Zhang F, Wang Y H and Li X Y. Viabilities of seismic ray impedance and elastic impedance for hydrocarbon-sand discrimination. Geophysics. 2012. 77(4): M39-M52

Zhang R, Sen M K and Srinivasan S. A prestack basis pursuit seismic inversion. Geophysics. 2013. 78(1): R1-R11

Zhang S X, Yin X Y and Zhang F C. Quasi fluid modulus for delicate lithology and fluid discrimination. SEG Technical Program Expanded Abstracts. 2010

Zhu X and McMechan G A. Elastic inversion of near- and postcritical reflections using phase variation with angle. Geophysics. 2012. 77(4): R149-R159

Zoeppritz K and Erdbebnenwellen V. On the reflection and penetration of seismic waves through unstable layers. Göttinger Nachrichten. 1919. 1: $66-84$

Zong Z Y, Yin X Y and Wu G C. Elastic impedance variation with angle inversion for elastic parameters. Journal of Geophysics and Engineering. 2012a. 9(3): 247

Zong Z Y, Yin X Y and Wu G C. AVO inversion and poroelasticity with P- and S-wave moduli. Geophysics. 2012b. 77(6): N29-N36

\section{Appendix A}

\section{Introduction of artificial neural network inversion}

The artificial neural network inversion was initially proposed by Růžek et al (2009). It is a kind of nonlinear direct inversion approach rather than optimization approach. It mainly contains the following steps.

\section{Problem initialization}

There is no need to allocate any specific models into the starting model population, even if doing so is easy. We just need to define the range for each model of the parameters,

$$
m_{i}^{\min }<m_{i}<m_{i}^{\max } \quad(i=1,2,3,4,5,6)
$$

The starting population of models is generated from the defined range with uniform probability. The number of models in the starting population $q$ is not very important because it will change during iterations. A suitable choice can be $30 \leq q \leq 60$. At each iteration, the current population of models $M^{B}=\left\{\mathbf{m}^{B}, \mathbf{d}^{B}, e r r^{B}\right\}$ is sorted according to the individual errors between the models and the candidate solution. The diameter of the population $R$ defines the size of a subspace, inside which the next population of models will be generated, and the index of the prediction function ip specifies the prediction method used for predicting the candidate solution, including linear regression $(i p=1)$, radial basis function network $(\mathrm{RBFN})(i p=2)$, and Kriging prediction $(i p=3)$. Both of these parameters can be tuned during the inversion, but in the beginning they are both set to 1 .

\section{Prediction of population and candidate solution}

There is a geometrical criterion in distinct iteration cycles before population predicting, one model is selected as the center of the population $\left(\mathbf{m}^{C}\right)$, and the other surrounding models are located randomly in the distance $R$ measured from the center of the population.

The prediction population is generated in such a way that the center is located close to the expected solution, and surrounding models are located randomly along the surface of a hypersphere with diameter $R$ and center $\mathbf{m}^{C}$. Provided both the diameter $R$ and the center $\mathbf{m}^{C}$ are known, new models of the predicting population can be obtained as follows. Firstly, the center of the population is considered as the first model. The matrix tensor $\mathbf{C}^{m}$ whose value on the principal diagonal line is defined as the square of the difference between the maximum and minimum of each model parameter can be decomposed using a Choleski decomposition as $\mathbf{C}^{m}=\mathbf{L} \quad \mathbf{L}^{\mathrm{T}}$. Secondly, a random six dimensional unit vector $\mathbf{g}$ is generated. Then, the proposed candidate model can be expressed as,

$$
\mathbf{m}^{g}=\mathbf{m}^{C}+R \mathbf{L} \cdot \mathbf{g}
$$

In any case when the candidate model $\mathbf{m}^{C}$ is outside the parametric hypercube, it is projected along the direction $\left(\mathbf{m}^{g}-\mathbf{m}^{C}\right)$ to the closest face of the parametric hypercube. The archive of already evaluated models is checked and the model $\left\{\mathbf{m}^{k}, \mathbf{d}^{k}, e r r^{k}\right\}$ is selected, and this model is the closest archive model to $\mathbf{m}^{g}$ and still not connected to the predicting population. We need to compute the distance $s^{g}$ between this model and the candidate model. If the distance $s^{g}$ is smaller than the distance between neighboring surrounding models, the model $\left\{\mathbf{m}^{k}, \mathbf{d}^{k}, e r r^{k}\right\}$ is connected to the population, otherwise, the candidate model $\mathbf{m}^{g}$ is evaluated and is connected to the predicting population and copied to the archive for future use. Finally, the second step made $q-1$ times to obtain a population of total size $q$.

The prediction population above can be used for estimating the solution (8) with the prediction algorithm. The prediction algorithm is implemented for the local approximation of the inverse mapping. Different prediction algorithms with different index ip can be selected to estimate the solutions, however the best solution is often to use different prediction algorithms even inside each individual inverse problem. Therefore, in our case, we use the prediction algorithm in a cyclic manner according to the variable $i p$.

(Edited by Hao Jie) 